\title{
BASIC SINGULARITIES IN THE THEORY OF INTERNAL WAVES WITH SURFACE TENSION
}

\author{
M. A. GORGUI and M. S. FALTAS \\ Department of Mathematics \\ Faculty of Science \\ Moharrem Bay \\ Alexandria, EGYPT \\ (Received September 6, 1984)
}

ABSTRACT. The study of linearized interface wave problems for two superposed fluids often involve's the consideration of different types of singularities in one of the two fluids. In this paper the line and point singularities are investigated for the case when each fluid is of finite constant depth. The effect of surface tension at the surface of separation is included.

KEY WORDS ANI PHRASES. Internal waves, surface tension, basic singularities. 1980 AMS SHABUECT CLASSIFICATION CODE. 76 B15, 76C10.

\section{INTRODUCTION .}

The study of internal waves in two fluids problems has attracted many authors in recent years. It is found useful to permit singularities of one type or another to occur as an idealisation of, or an approximation to certain physical situations. Problems dealing with the generation of waves at the interface of two non-mixing fluids involve the consideration of singularities of different types in the fluids. In the case when bodies are present, waves may either be generated by the movement of the body or reflected from it. The two cases are essentially the same and the resulting motion can be described by the use of these singularities in a suitable way. For example, Gorgui [1] has investigated into these waves using a distribution of sources on the surface of the body.

The different types of singularities that can be used in two fluids problems have been presented by Gougui and Kassem [2] and Kassem [3] ; in both the effects of surface tension are neglected. In [2], the authors considered the cases when the lower fluid is of finite constant depth and the upper fluid is of infinite height. While in [3] the author considered the cases when the two superposed fluids are both of finite thickness and obtained the potentials for motions resulting from multipoles submerged in one of the two fluids.

In this paper we give a complete survey for the basic line and point singularities when the superposed fluids are as in [3] of finite constant thickness confined between two rigid horizontal planes but we here take surface tension into consideration. 
In the two dimensional motion, the line singularities considered are wave sources and multipoles singularities. Restriction is made to symmetric (or vertical) multipoles, but the corresponding antisymetric (or horizontal) multipoles can be found similarly. For axisymmetric motion, the point singularities considered are multipole singularilies. These time harmonic singularities are described by harmonic potential functions which satisfy two 1 inearised conditions at the surface of separation, and uniquenes: is ensured by requiring that there are only outgoing waves in the far field. The method used is an extension of that used in [2] or 131 . The results obtained by Rhodes-Robinson [4], Gorrui and Kassem [2] and Kassem [3] can be deduced as special casis.

2. STALE:IENT OF THE PRUBLFN.

Wo are concerned with the irrotational, incompressible and inviscid motion of the two supernosed non-mixin: fluids under the action of gravity and surface tension. Each fluid is of infinite horizantal extent. Taking the ori!, in 0 at the mean level of the interface and the axis oy pointing vertically downwards into the lower fluid, let the two fluid'; be confined between rigid horizontal planes $y=h, y=-h$ '. The motion is simple harmonic with a small amplitude and angular frequency $\sigma$; it is due to an oscillating singularity in one of the two fluids. In two dimensional motion we consider the singularity is either a line wave source or multipole and in axisymmetric motion it is a point multipole. In each case, the velocity potentials of the lower and upper tluids are simple harmonic with period $\frac{2 \pi}{\sigma}$ and it is more convenient to use the complex valued potentials $\phi e^{-i \sigma t}, \phi e^{-i \sigma t}$, of which the actual velocity potentials are the rual parts.

These potentials satisfy a boundary value problem in which

$$
\nabla^{2} \emptyset=0, \nabla^{2} \emptyset^{\prime}=0
$$

in the re,ions occupied by the fluids, except at the singularity;

$$
\begin{array}{lll}
\frac{\partial \eta}{\partial y}=0 & \text { on } & y=h, \\
\frac{\partial \emptyset^{\prime}}{\partial y}=0 & \text { on } & y=-h^{\prime},
\end{array}
$$

and the 1 inearized boundary conditions

$$
\begin{aligned}
& \frac{\partial \emptyset}{\partial y}=\frac{\partial \emptyset^{\prime}}{\partial y^{\prime}}, \\
& K \emptyset+\frac{\partial \emptyset}{\partial y}-s\left(K \emptyset^{\prime}+\frac{\partial \emptyset}{\partial y}\right)+M \frac{\partial^{3} \emptyset}{\partial y^{3}}=0
\end{aligned} \quad \text { on } y=0
$$

where $K=\frac{\sigma^{2}}{g}, \quad M=\frac{T}{\rho g}, g$ is the gravity, $T$ is the surface tension, $\rho$ and $s \rho$ $(s<1)$ are the densities of the lower and upper fluids respectively. These conditions are applied for each singularity considered. They are supplemented by the two general limiting conditions that $\emptyset$ or $\emptyset^{\prime}$ behaves like a typical singular harmonic function near the singularity and the radiation condition that both functions represent outgoing waves in the far field.

\section{SUBMI:RGED LINE SINGiULARITIES.}

Without loss of generality, the line singularity is place at the point (o, $\pm n)$ We consider only singularities symmetric in $x$ - namely, a wave source and vertical 
multipoles. We define polar coordinates $(R, \theta)$ in the $x y-$ plane based at the singularity position by the equations

$$
x=R \sin \theta, y \pm n=R \cos \theta
$$

according as the singularity is in the lower or upper fluid, so that $R$ denotes the distance from the singularity.

(i) Wave source singularities

Here $\emptyset$ and $\emptyset^{\prime}$ arc solutions of the boundary - value problem stated above with $\emptyset$ having a logarithmic singularity at the source. If the source is at (o, $\eta)$ then

$$
\emptyset \sim \log R \text { as } R=\left[x^{2}+(y-n)^{2}\right]^{\frac{1}{2}} \rightarrow 0
$$

Let

$$
\begin{aligned}
& \emptyset=\log R+\alpha \log R^{\prime}+\int_{0}^{\prime \prime}[s f(k)+\{\Lambda \cosh k(h-y)+B \sinh k y\} \cos k x] d k, \\
& \emptyset^{\prime}=\alpha^{\prime} \log R+\int_{0}^{\infty}\left[f(k)+\left\{A^{\prime} \cosh k\left(h^{\prime}+y\right)+B^{\prime} \sinh k y\right\} \cos k x\right] d k,
\end{aligned}
$$

where $R^{\prime}=\left[x^{2}+(y+n)^{2} 1^{\frac{1}{2}}\right.$ is the distance from the image point $(0,-n)$. It is obvious that $\emptyset, \emptyset^{\prime}$ as given above are harmonic. We choose $\alpha, \alpha^{\prime}, f(k), A(k)$, $B(k), A^{\prime}(k), B^{\prime}(k)$ such that the two integrals converge and the boundary conditions on $y=h, y=-h^{\prime}$ and $y=o$ are satisfied.

Under suitable conditions concerning differentiation under the integral sign and using the relations

$$
\frac{\partial}{\partial y}(\log R)=\frac{\cos }{R} \theta=\left\{\begin{array}{l}
\int_{0}^{\infty} e^{-k(y-n)} \cos k x d k, y>n, \\
-\int_{0}^{\infty} e^{k(y-n)} \cos k x d k, y<n,
\end{array}\right.
$$

the conditions (2.2), (2.3) are satisfied if

$$
B=-\frac{e^{-k(h-n)}+\alpha e^{-k(h+n)}}{k \cosh k h}, \quad B^{\prime}=\frac{\alpha^{\prime} e^{-k\left(h^{\prime}+n\right)}}{k \cosh k h^{\prime}},
$$

and the interface conditions are sacisfied if

$$
1+\alpha-s \alpha^{\prime}=0 \text {, }
$$

$A \sinh k h+A^{\prime} \sinh k h^{\prime}=\frac{\alpha+\alpha^{\prime}-1}{k} e^{-k n}+\left(B-B^{\prime}\right)$,

$c A \cosh k h-A^{\prime}\left[s c \cosh k h^{\prime}-k\left(1+\beta k^{2}\right) \sinh k h^{\prime}\right]=\left(1+\beta k^{2}\right)\left(\alpha^{\prime} e^{-k n}-k^{\prime}\right)$, from which we have

$\Delta A=\alpha^{\prime}\left(1+\beta k^{2}\right) e^{-k n} \sinh ^{2} k h^{\prime} \operatorname{sech} k h+\left[\frac{s c}{k} \cosh k h^{\prime}-\left(1+\beta k^{2}\right) \sinh k h^{\prime}\right] \frac{F(k)}{\cosh k h^{\prime}}$, $\Delta A^{\prime}=-\alpha^{\prime}\left(1+\beta k^{2}\right) e^{-k n} \sinh k h \tanh k h^{\prime}+\frac{c F(k)}{k}$,

where

$$
\begin{gathered}
F(k)=\left(\alpha+\alpha^{\prime}-1\right) e^{-k n} \cosh k h-e^{-k(h-n)}-\alpha e^{-k(h+\eta)} \\
-\alpha^{\prime} \epsilon^{-k\left(h^{\prime}+\eta\right)} \cosh k h \operatorname{sech} k h^{\prime},
\end{gathered}
$$

$\Delta(k)=c\left(\cosh k h \sinh k^{\prime}+s \cosh k^{\prime} \sinh k h\right)-k\left(1+\beta k^{2}\right) \sinh k h \sinh ^{\prime} h^{\prime},(3.2)$

$$
c=\frac{K}{1-S}, \quad B=\frac{M}{1-S} \text {. }
$$


Since $F(0)=-2$, therefore, the integrals involved in the assumed expressions for $A, A^{\prime}$ converge if we choose $f(k)$ such that $k \Delta f(k)=2 c+0\left(k^{2}\right)$ in the neighbourhood of $k=0$ and if $\frac{\mathrm{dF}(0)}{\mathrm{dk}}=0$, i.e.,

$$
h(1+\alpha)+h^{\prime} \alpha^{\prime}=0
$$

Hence

$$
\begin{gathered}
\alpha^{\prime}=0 \quad, \alpha=-1, \\
F(k)=-2 \cosh k(h-n), \\
B=-2 \frac{e^{-k h} \frac{\sinh k n}{k} \cosh k h}{B^{\prime}=0,} \\
A=2\left[\left(1+B k^{2}\right) \sinh k h^{\prime}-\frac{s c}{k} \cosh k h^{\prime}\right] \frac{\cosh k(h-n)}{\Delta \cosh k h}, \\
A^{\prime}=-2 c \frac{\cosh k(h-\eta)}{k \Delta}
\end{gathered}
$$

The condition imposed on $f(k)$ does not specify it completely. This introduces no difficulty since it is the velocities in which we are really interested. It is found convenient to take $f(k)=\frac{2 c}{k \Delta}$.

Now, $\Delta(k)$ has a simple zero at $k=m$, say, on the real axis of $k$. This introduces simple poles for the integrals in $\emptyset$, $\emptyset^{\prime}$. Below this pole we make an indentation of the contours of the integrations. Substituting in the above assumed forms for $A, A^{\prime}, B, B^{\prime}$ we get

$\emptyset=\log \frac{R}{R^{\prime}}+2 f_{0}^{\infty}\left[\frac{c s}{k \Delta}+\left[\left\{k\left(1+\beta k^{2}\right) \sinh k h^{\prime}-s c \cosh k h^{\prime}\right\} x\right.\right.$

, $\left.\left.\frac{\cosh k(h-\eta)}{k \Delta \cosh k h} \cosh k(h-y)-\frac{e^{-k h} \sinh k n}{k \cosh k h} \sinh k y\right] \cos k x\right] d k$,

$$
\emptyset^{\prime}=2 c f_{0}^{\infty} \frac{1}{k}\left[1-\cosh k(h-n) \cosh k\left(h^{\prime}+y\right) \cos k x\right] d k \text {. }
$$

Two other alternative forms which will be useful in the subsequent work are

$$
\begin{aligned}
& \emptyset=\log R+(2 s-1) \log R^{\prime}+2 f_{0}^{\infty} \frac{\delta}{\Delta}\left(1+\beta k^{2}\right) \sinh ^{2} k h^{\prime} \cosh k(k-n) x \\
& \quad \times \cosh k(h-y) \cos k x d k+2 s \int_{0}^{\infty} \frac{1}{k}\left[\delta-e^{-k h^{\prime}}+\right. \\
& \left.\left.\Gamma e^{-k(y+n)}-\delta \cosh k(h-n) \frac{\cosh k h^{\prime}}{\cosh k h} \cosh k(h-y)\right] \cos k x\right] d k \\
& \quad-2 \int_{0}^{\infty} \frac{e^{-k h} \sinh k n \frac{\sinh k y}{\cosh k h} \cos k x d k,}{\emptyset^{\prime}=2 \log R-2 f_{0}^{\infty} \frac{\delta}{\Delta}\left(1+\beta k^{2}\right) \sinh k h \sinh k h^{\prime} \cosh k(h-n) x} \\
& \times \cosh k\left(h^{\prime}+y\right) \cos k x d k+2 \int_{0}^{\infty} \frac{1}{k}\left[\delta-e^{-k h^{\prime}}+\left[e^{-k(n-y)}-\right.\right. \\
& \left.\left.\quad-\delta \cosh k(h-\eta) \cosh k\left(h^{\prime}+y\right)\right] \cos k x\right] d k,
\end{aligned}
$$

where $\delta^{-1}=\cosh k h \sinh k^{\prime}+s \cosh k^{\prime} \sinh k h$.

In the above expressions we neglected the constants

$2 s \log h^{\prime}+2 s \int_{0}^{\infty} \frac{\delta}{\Delta}\left(1+\beta k^{2}\right) \sinh k^{\prime} \sinh k h d k$ and

$2 \log h^{\prime}+2 \int_{0}^{\infty} \frac{\delta}{\Delta}\left(1+\beta k^{2}\right) \sinh k h \sinh k h^{\prime} d k$ in $\emptyset$, $\emptyset^{\prime}$ respectively. 
By putting $2 \cos k x=e^{i k|x|}+e^{-i k|x|}$, and rotating the contours in the indented integrals in $\emptyset, \emptyset^{\prime}$ into contours in the first and fourth quadrants so that we must include the residue term at $k=m$, these integral tend to

$$
-C\left(o ; h, h^{\prime}\right) \frac{\cosh m(h-y)}{m \sinh m h} e^{i m|x|}, C\left(o ; h, h^{\prime}\right) \frac{\cosh m\left(h^{\prime}+y\right)}{m \sinh m^{\prime}} e^{i m|x|}
$$

as $|x| \rightarrow \infty$, where

$$
C\left(n ; h, h^{\prime}\right)=-\frac{\pi i c m^{n}}{n !} \frac{\left[e^{-m(h-n)}+(-1)^{n} e^{m(h-n)}\right] \sinh ^{2} m h^{\prime} \sinh m h}{\left(1+3 h m^{2}\right) \sinh ^{2} m h \sinh ^{2} m h^{\prime}+c\left(h \sinh ^{2} m h^{\prime}+h^{\prime} \sinh \sinh ^{2}\right)}
$$

When the source is at $(0,-n)$ in the upper field, we have

$$
\emptyset^{\prime} \sim \log R \text { as } R=\left[x^{2}+(y+n)^{2}\right]^{\frac{1}{2}} \rightarrow 0
$$

Assume the forms

$$
\begin{array}{r}
\emptyset=\alpha \log R+\int_{0}^{\prime \alpha}[s f(k)+\{A \cosh k(h-y)+B \sinh k y\} \cos k x] d k, \\
\emptyset^{\prime}=\log R+\alpha^{\prime} \log R^{\prime}+\int_{0}^{\infty}\left[f(k)+\left\{A^{\prime} \cosh k\left(h^{\prime}+y\right)+B^{\prime} \sinh k y\right\}\right. \\
\cdot \cos k x] d k,
\end{array}
$$

where $R^{\prime}=\left[x^{2}+(y-n)^{2}\right]^{\frac{1}{2}}$ is the distance from the image point (o, $\left.n\right)$ and proceed as above to get the potentials

$$
\begin{aligned}
& \emptyset=2 \operatorname{cs} f_{0}^{\infty} \frac{1}{k \Delta}\left[1-\operatorname{cosin} k\left(h^{\prime}-n\right) \cosh k(h-y) \cos k x\right] d k, \\
& \emptyset^{\prime}=\log \frac{\mathrm{R}}{\mathrm{R}^{\prime}}+2 \underset{0}{f^{\infty}} \dot{i} \frac{\mathrm{c}}{\mathrm{k} \Delta}+\left[\left(\mathrm{k}\left(1+\beta \mathrm{k}^{2}\right) \sinh \mathrm{kh}-\mathrm{c} \cosh \mathrm{kh}\right) \frac{\left.\cosh \mathrm{k}^{\prime} \mathrm{h}^{\prime}-\eta\right)}{\mathrm{k} \Delta \cosh ^{\prime} \mathrm{kh}^{\prime}} \lambda\right. \\
& \left.\left.x \cosh k\left(h^{\prime}+y\right)+e^{-k h^{\prime}} \frac{\sinh k \eta}{k \cosh k h^{\prime}} \sinh k y\right] \cos k x\right\lrcorner d k,
\end{aligned}
$$

and the other two alternatives forms are

$$
\begin{aligned}
\emptyset= & 2 s \log R-2 s f_{0}^{\infty} \frac{\delta}{\Delta}\left(1+\beta k^{2}\right) \sinh k h \sinh k h^{\prime} \cosh k\left(h^{\prime}-n\right) x \\
& \times \cosh k(h-y) \cos k x d k+2 s \int_{0}^{\infty} \frac{1}{k}\left(\delta-e^{-k h^{\prime}}\right)+ \\
& +\left[e^{-k(y+n)}-\delta \cosh k\left(h^{\prime}-n\right) \cosh k(h-y)\right] \cos k x d k, \\
\emptyset^{\prime}= & \log R R^{\prime}+2 s f_{0}^{x} \frac{\delta}{\Delta}\left(1+B k^{2}\right) \sinh k h \cosh k\left(h^{\prime}-n\right) \cosh \left(h^{\prime}+y\right) \times \\
\times & \cosh k\left(h^{\prime}+y\right) \cos k x d k+2 \int_{0}^{\infty} \frac{1}{k}\left[\left(\delta-e^{-k h^{\prime}}\right)+\left[e^{-k(n-y)}\right.\right. \\
& \left.\left.-\delta \cosh k\left(h^{\prime}-n\right) \frac{\cosh k h}{\cosh k h^{\prime}} \cosh k\left(h^{\prime}+y\right)\right] \cos k y\right] d k+ \\
& +2 \int_{0}^{\infty} e^{-k h^{\prime}} \frac{\sinh k n s i n h k y}{k} \frac{\cosh k h^{\prime}}{\cos k x d k} \cdot
\end{aligned}
$$

These potentials have the outgoing waves

$$
\begin{aligned}
& -C\left(o ; h^{\prime}, h\right) \frac{s \cosh m(h-y)}{\sinh m h} e^{i m|x|}, \\
& C\left(o ; h^{\prime}, h\right) \frac{s \cosh m\left(h^{\prime}+y\right)}{m \sinh m^{\prime}} e^{i m|x|,}
\end{aligned}
$$

as $|\mathrm{x}| \rightarrow \infty$ where $C\left(\mathrm{n} ; \mathrm{h}, \mathrm{h}^{\prime}\right)$ is given by $(3.7)$.

(ii) Multipoles singularities

Here $\emptyset, \emptyset^{\prime}$ are harmonic in the regions occupied by the two fluids except at the singularity. In the neighbourhood of this point 


$$
\emptyset \sim \frac{\cos (\mathrm{n}+1) \theta}{\mathrm{R}^{\mathrm{n}}+1}, \mathrm{n}=0,1,2, \ldots
$$

We consider first the case when the singularity is at $(0, n)$ in the lower fluid. We try as solutions

$$
\begin{aligned}
& \emptyset=\frac{\cos (n+1)}{R^{n+1}}-\int_{0}^{\infty}[A \cosh k(h-y)+B \sinh k y] \cos k x d k, \\
& \emptyset^{\prime}=\int_{0}^{\infty} A^{\prime} \cosh k\left(h^{\prime}+y\right) \cos k x d k,
\end{aligned}
$$

and use the representations

$$
\frac{\cos (n+1) \theta}{R^{n+1}}=\left\{\begin{array}{l}
\frac{(-1)^{n+1}}{n !} \int_{0}^{\infty} k^{n} e^{k(y-n)} \cos k x d k, y<n, \\
\frac{1}{n !} \int_{0}^{(x)} k^{n} e^{-k(y-n)} \cos k x d k, y>n .
\end{array}\right.
$$

Conditions $(2.2)-(2.4)$ are satisfied if

$$
B=\frac{k^{n} e^{-k(h-n)}}{n ! \cosh k h} \text {, }
$$

$A \sinh k h+A^{\prime} \sinh k h^{\prime}=B+\frac{(-1)^{n}+1}{n !} k^{n} e^{-k n}$,

$-c A \cosh k h+\left[s c \cosh k h^{\prime}-k\left(1+B k^{2}\right) \sinh k h^{\prime}\right] A^{\prime}=\frac{(-1)^{n+1}}{n !} c k^{n} e^{-k n}$.

These determine $A, B, A^{\prime}$, which when substituted in the above assumed forms, give

$$
\begin{aligned}
& \emptyset=\frac{\cos }{R^{n}} \frac{(n+1) \theta}{+1}+\frac{1}{n !} \int_{0}^{\infty} k^{n} e^{-k(h-n)} \frac{\sinh k y}{\cosh k h} \cos k x d k \\
&+\frac{1}{n !} \int_{0}^{\infty} \frac{k^{n}}{\Delta} P(n) \cos k x d k, \\
& \emptyset=\frac{c}{n !} \int_{0}^{\infty} \frac{k^{n}}{\Delta}\left[e^{-k(h-n)}+(-1)^{n+1} e^{k(h-n)}\right] \cosh k\left(h^{\prime}+y\right) \cos k x d k,
\end{aligned}
$$

where

$$
\begin{gathered}
P(n)=(-1)^{n+1}\left[c\left(s \cosh k^{\prime}-\sinh k h^{\prime}\right)-k\left(1+\beta k^{2}\right) \sinh k h^{\prime}\right] e^{-k n} \\
+\left[\operatorname{cscosh} k h^{\prime}-k\left(1+\beta k^{2}\right) \sinh k h^{\prime}\right] \frac{e^{-k(h-n)}}{\cosh k h},
\end{gathered}
$$

and $\Delta$ is given by equation (3.2).

As $|x| \rightarrow \infty$, we have the outgoing waves

$\emptyset \sim(n+1) C\left(n+1 ; h, h^{\prime}\right) \frac{\cosh m(h-y)}{m \sinh m h} e^{i m|x|}$,

$$
\phi \sim-(n+1) C\left(n+1 ; h, h^{\prime}\right) \frac{\cosh m\left(h^{\prime}+y\right)}{m \sinh m h^{\prime}} e^{i m|x|,}
$$

where $C\left(n ; h, h^{\prime}\right)$ is given by equation (3.7).

If the singularity is at $(0,-n)$ in the upper fluid we try as solutions $\phi=\int_{0}^{\infty} A \cosh k(h-y) \cos k x d k$, $\phi^{\prime}=\frac{\cos (\mathrm{n}+1) \theta}{\mathrm{R}^{\mathrm{n}-1}}+\int_{0}^{\infty}\left[\mathrm{A}^{\prime} \cosh \mathrm{k}^{\prime}\left(\mathrm{h}^{\prime}+\mathrm{y}\right)+\mathrm{B}^{\prime} \sinh \mathrm{ky}\right] \cos \mathrm{kx} d \mathrm{k}$;

where $R=\left[x^{2}+(y+n)^{2}\right]^{\frac{1}{2}}$.

Proceeding as above leads to

$$
\phi=\frac{s c}{n !} \stackrel{f}{0}_{0}^{\infty} \frac{k^{n}}{\Delta}\left[e^{k\left(h^{\prime}-n\right)}+(-1)^{n+1} e^{-k\left(h^{\prime}-n\right)}\right] \cos k(h-y) \cos k x d k,
$$




$$
\begin{aligned}
& \phi^{\prime}=\frac{\cos (n+1) \theta}{R^{n+1}}-\frac{(-1)^{n+1}}{n !} \int_{0}^{\infty} \frac{k^{n} e^{-k\left(h^{\prime}-n\right)}}{\cosh k h^{\prime}} \sinh k y \cos k x d k \\
& +\frac{1}{n !} \stackrel{\infty}{f}_{0}^{\infty} \frac{k^{n}}{\Delta} Q(n) \cosh k\left(h^{\prime}+y\right) \cos k x d k \text {, }
\end{aligned}
$$

where

$$
\begin{aligned}
Q(n) & =\left[c(\cosh k h-s \sinh k h)-k\left(1+\beta k^{2}\right) \sinh k h\right] e^{-k n} \\
& +(-1)^{n+1}\left[c \cosh k h-k\left(1+\beta h^{2}\right) \sinh k h\right] \frac{e^{-k\left(h^{\prime}-n\right)}}{\cosh k h^{\prime}} .
\end{aligned}
$$

The contours of the integrals in (3.15), (3.16) are indented below the simple pole at $k=m$ to give the outgoing waves

$$
\begin{aligned}
& \left.\phi \sim s(n+1) C(n+1), h^{\prime}, h\right) \frac{\cosh m(h-y)}{n \sinh m h} e^{i m|x|}, \\
& \phi^{\prime} \sim-s(n+1) C\left(n+1 ; h^{\prime}, h\right) \frac{\cosh \left(h^{\prime}+y\right)}{n \sinh m h^{\prime}} e^{i m|x|},
\end{aligned}
$$

as $|\mathbf{x}| \rightarrow \infty$.

4. SUBMERGED POINT SINGULARITIES.

We now define cylindrical polar coordinates $(r, \psi, y)$ with the origin 0 at the surface separating the two fluids and the $y$-axis pointing vertically downwards. We also define spherical polar coordinates $(R, \theta, \psi)$ based at the singularity taken at $(0, \pm n)$ by the equations

$$
r=R \sin \theta, y \mp n=R \cos \theta
$$

We consider only point singularities for which $0 y$ is an axis of symmetry so that the velocity potentials $\phi, \phi^{\prime}$ are independent of $\psi$.

When the singularity is at $(o, n)$ in the lower fluid the boundary value problem for $\phi, \phi^{\prime}$ is given by $(2.1)-(2.4)$ supplemented by the limiting condition

$$
\phi \sim \frac{P_{n}(\cos \theta)}{R^{n+1}} \text { as } R=\left[r^{2}+(y-n)^{2}\right]^{\frac{1}{2}} \rightarrow 0, n=0,1,2, \ldots
$$

If we try as solutions

$$
\begin{aligned}
& \phi=\frac{P_{n}(\cos \theta)}{R^{n+1}}+\int_{0}^{\infty}[A \cosh k(h-y)+B \sinh k y] J_{0}(k x) d k, \\
& \phi^{\prime}=\int_{0}^{\infty} A^{\prime} \cosh k\left(h^{\prime}+y\right) J_{0}(k r) d k,
\end{aligned}
$$

and using the representations

$$
\frac{P_{n}(\cos \theta)}{R^{n+1}}=\left\{\begin{array}{l}
\frac{(-1)^{n}}{n !} \int_{0}^{\infty} k^{n} e^{k(y-n)} J_{0}(k r) d k, y<n, \\
\frac{1}{n !} \int_{0}^{\infty} k^{n} e^{-k(y-n)} J_{0}(k r) d k, y>n,
\end{array}\right.
$$

conditions (2.2), (2.4) are satisfied if

$$
B=\frac{k^{n} e^{-k(y-n)}}{n ! \cosh k h} \text {, }
$$

$A \sinh k h+A^{\prime} \sinh k h^{\prime}=B+\frac{(-1)^{n}}{n !} k^{n} e^{-k n}$,

$-c A \cosh k h+\left[s c \cosh k h^{\prime}-k\left(1+B k^{2}\right) \sinh k h^{\prime}\right] A^{\prime}=\frac{(-1)^{n}}{n !} c k^{n} e^{-k n}$.

Solving these equations and substituting, we obtain the expressions 


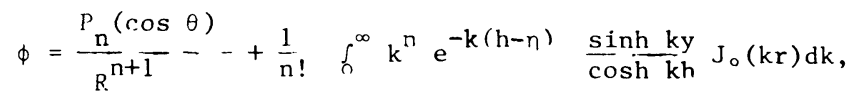

$$
\begin{aligned}
& \frac{1}{n !} f_{0}^{\infty} \frac{k^{n}}{\Delta} P(n-1) \cosh k(h-y) J_{0}(k r) d k \text {, } \\
& \phi^{\prime}=\frac{c}{n !} f_{0}^{\infty} \frac{k^{n}}{\Delta}\left[e^{-h(h-n)}+(-1)^{n} e^{k(h-n)}\right] \cosh k\left(h^{\prime}+y\right) J_{0}(k r) d k,
\end{aligned}
$$

where $\Delta$ is given by (3.2), $P(n)$ is given bv (3.14) and as before the contour of integration is indented below the simple root $k=m$ of $\Delta=0$ on the positive real k-axis, which encures that the radiation conditions are satisfied. For, by putting

$$
2 J_{0}(k r)=H_{0}^{(1)}(k r)+H_{0}^{(2)}(k r),
$$

rotating the contours in each integral into contours in the first and fourth quadrants (where $\mathrm{H}_{r}^{(1)}(\mathrm{kr}), \mathrm{H}_{0}^{(2)}(\mathrm{kr})$ are respectively small) and including the residue term at $k=m$, we obtain the diverging waves

$$
\begin{aligned}
& \phi \sim \mathrm{C}\left(\mathrm{n} ; \mathrm{h}, \mathrm{h}^{\prime}\right) \frac{\cosh }{\sinh } \frac{\mathrm{m}(\mathrm{h}-\mathrm{y})}{\mathrm{mh}} \mathrm{H}_{\circ}^{(1)}(\mathrm{mr}), \\
& \phi^{\prime} \sim-C\left(n ; h, h^{\prime}\right) \frac{\cosh m\left(h^{\prime}+y\right)}{\sinh m h^{\prime}} H_{o}^{(1)}(m r) \text {, }
\end{aligned}
$$

as $r \rightarrow \infty$, where $C\left(n ; h, h^{\prime}\right)$ is given by equation (3.7).

In a similar manner we calculate the velocity potential when the multipole singularity is at $(0,-n)$ in the upper liquid. These are

$$
\begin{aligned}
\phi= & \frac{s c}{n !} f_{0}^{\infty} \frac{k^{n}}{\Delta}\left[e^{k\left(n^{\prime}-n\right)}+(-1)^{n} e^{\left.-k\left(h^{\prime}-n\right)\right] \cosh k(h-y) J_{0}(k r) d k}\right. \\
\phi^{\prime}= & \frac{P_{n}(\cos \theta)}{R^{n+1}}-\frac{(-1)^{n}}{n !} \int_{0}^{\infty} \frac{k^{n} e^{-k\left(h^{\prime}-n\right)}}{\cosh k^{\top}} \sinh k y J_{0}(k r) d k \\
\quad & \quad \frac{1}{n !} x_{0}^{\infty} \frac{k^{n}}{\Delta}-Q(n-1) \cosh k\left(h^{\prime}+y\right) \cosh k^{\prime}\left(h^{\prime}+y\right) J_{0}(k r) d k
\end{aligned}
$$

where $R=\left[r^{2}+(y+n)^{2}\right]^{\frac{1}{2}}$ and $Q(n)$ is given by (3.17). This motion has the diverging cylindrical waves

$$
\begin{aligned}
& \phi \sim C\left(n ; h^{\prime}, h\right) \frac{s \cosh m(h-v)}{a i n h m h} H_{0}^{(1)}(m r), \\
& \phi^{\prime} \sim C\left(n ; h^{\prime}, h\right) \frac{s \cosh m\left(h^{\prime}+y\right)}{\sinh m h} H_{0}^{(1)}(m r),
\end{aligned}
$$

as $r \rightarrow \infty$.

5. SUBMERGED SINGULARITIES; THE LOWER FLUID IS OF FINITE CONSTANT DEPTH AND THE UPPER IS UNBOUNDED.

A statement of the boundary-value problems for the velocity potentials $\phi$, $\phi^{\prime}$ for the different types of singularities can be easily written down. These of the present case are similar to the corresponding ones treated in sections 3 and 4 with condition (2.3) replaced by

$$
\nabla \phi^{\prime} \rightarrow 0 \text { as } \mathrm{y} \rightarrow-\infty
$$

and the radiation condition taking the simplet forms

$$
\begin{aligned}
& \phi \sim C \cosh m(h-y) e^{i m|x|}, \\
& \phi^{\prime} \sim-C e^{m y} e^{i m|x|},
\end{aligned}
$$

as $|x| \rightarrow \infty$ for line singularities, and the forms 


$$
\begin{aligned}
& \phi \sim C \cosh m(h-y) H^{(1)}(m r), \\
& \phi^{\prime} \sim-C e^{m y}{ }_{H}^{(1)}(m r),
\end{aligned}
$$

as $r \rightarrow \infty$ for point singularities, where $C$ is a constant multiplier and $m$ is a simple root of the equation $\Delta=0$ where now

$\Delta=c(\cosh k h+s \sinh k h)-k\left(1+\beta k^{2}\right) \sinh k h$.

The determination of $\phi, \phi^{\prime}$ for each singularity can be carried out independently. This was done by the second author [5], where he assumed $\phi, \phi^{\prime}$ to have the appropriate forms. They may also be determined by letting $h^{\prime} \rightarrow \infty$ in the formulae obtained in the above sections. The velocity potentials for the different cases are as follows:

(a) Line singularities

(i) For a wave source at $(0, n)$ in the lower fluid,

$\phi=\log R+(2 s-1) \log R^{\prime}+2 f_{0}^{\infty} \frac{\delta}{\Delta}\left(1+\beta k^{2}\right) \cosh k(h-\eta) \cosh k(h-y) \times$

$x \cos k x d k+\int_{0}^{\infty} \frac{2}{k}\left[s e^{-k(y+n)}-e^{-k h} \frac{\sinh k n \sinh k y}{\cosh k h}\right.$

$\left.-\frac{s \delta \cosh k(h-\eta) \cosh k(h-y)}{\cosh k h}\right] \cos k x d k$,

$\phi^{\prime}=2 \log k-2 f_{0}^{\infty} \frac{\delta}{\Delta}\left(1+B k^{2}\right) \sinh k h \cosh k(h-n) e^{k y} \cos k x d k$

$+\int_{0}^{\infty} \frac{2}{k}\left[e^{-k n}-\delta \cosh k(h-\eta)\right] e^{k y} \cos k x d k$

where now $\delta^{-1}=\cosh k h+s \sinh k h$. The path of integration is along $\operatorname{Im}(k)=0$ o to ${ }^{\infty}$, indented below the simple pole at $\mathrm{k}=\mathrm{m}$.

These potentials have the outgoing waves

$-C(o) \frac{\cosh m(h-y)}{m \sinh m h} e^{i m|x|}, \quad C(o) \frac{e^{m y}}{m} e^{i m|x|}$, as $|x| \rightarrow \infty$, where

$C(n)=\frac{\pi i c}{n !} m^{n} \frac{\left[e^{-m(h-n)}+(-1)^{n} e^{m(h-n)}\right] \sinh m h}{h c+\left(1+3 \beta m^{2}\right) \sinh ^{2}} \frac{m h}{\text {, }}$

The above velocity potentials can be written in slightly different forms suitable for use in the next section. These are

$\phi=\log R-\frac{1-\underline{s}}{1+s} \log R^{\prime}+2 \varphi_{0}^{\infty} \frac{\delta}{\Delta}\left(1+\beta k^{2}\right) \cosh k(h-n) \cosh k(h-y) \cos k x d k$ $+\frac{2}{1+s} \int_{0}^{\infty} e_{k}^{-k h}\left[s^{2}-\delta(\sinh k n+s \cosh k n)(\sinh k y+s \cosh k y) \cos k x\right] d k(5.5)$

$\phi^{\prime}=\frac{2}{1+s} \log R-2 f_{0}^{\infty} \frac{\delta}{\Delta}\left(1+\beta k^{2}\right) \sinh k h \cosh k(h-n) e^{k y} \cos k x d k+\frac{2}{1+s} \int_{0}^{\infty} \frac{e^{-k h}}{k} x$

$\times\left[s-\delta(\sinh k n+s \cosh k n) e^{k y} \cos k x\right] d k$.

When the wave source is at $(0,-n)$ in the upper fluid,

$\phi=2 s \operatorname{sog} R-2 s f_{0}^{\infty} \frac{\delta}{\Delta} e^{-k n}\left(1+B k^{2}\right) \sinh k h \cosh k(h-y) \cos k x d k+2 s \int_{0}^{\infty} e_{k}^{-k h} x$

$x\left[e^{-k y}-\delta \cosh k(h-y)\right] \cos k x d k$ 
$\phi^{\prime}=\log R R^{\prime}+2 s f_{0}^{\infty} \frac{\delta}{\Delta}\left(1+B k^{2}\right) \sinh ^{2} k h e^{-k(n-y)} \cos k x d k+2 s \int_{0}^{\infty} \frac{\delta}{k} \sinh k h x$

$x e^{-k(n-y)} \cos k x d k$.

or as in the previous case,

$\phi=\frac{2 s}{1+s} \operatorname{lng} R-2 s t_{t}^{\infty} \frac{\delta}{\Delta} e^{-k n}\left(1+\beta k^{2}\right) \sinh k h \cosh k(h-y) \cos k x d k+\frac{2 s}{1+s} \int_{0}^{\infty} e^{-k h} \frac{k^{-}}{x}$

$\left.\wedge \mid s-\delta e^{-k n}(\sinh k y+s \cosh k y) \cos k x\right] d k$,

$\phi^{\prime}=\operatorname{lng} R+\frac{1-s}{1+s} \log R^{\prime}+2 s f_{0}^{\infty} \delta\left(1+\beta k^{2}\right) e^{k(y-n)} \sinh ^{2} k h \cos k x d k+\frac{2 s}{1}-\bar{s} \int_{0}^{\infty} \underline{e}_{k}^{-k h}-x$

$x\left(1-\delta e^{k(y-n)} \cos k x\right) d k$

These potentials have the outgoing waves

$$
-C^{\prime}(0) \frac{\cosh m(h-y)}{m} \sinh \frac{m h}{i m|x|}, \quad C^{\prime}(0) \frac{e^{m y}}{m}-e^{i m|x|},
$$

as $|x| \rightarrow \infty$, where

$$
C^{\prime}(n)=-2 \pi-\frac{i s c m^{n}}{n !} \quad \frac{e^{-m n s i n h^{2} m h}}{h c+\left(1+3 B m^{2}\right) s i n h^{2} m h}
$$

(ii) Multipoles singularities

When the singularity is at $(0, n)$ in the lower fluid,

$$
\begin{aligned}
& \phi=\frac{\cos (n+1) \theta}{R^{n+1}}+\frac{1}{n !} \int_{0}^{\infty} k^{n} e^{-k(h-n)} \frac{\sinh k y}{\sinh k h} \cos k x d k-\frac{1}{n !} f^{\infty} k^{n}\left[(-1)^{n+1} x\right. \\
& \begin{array}{r}
\left.\left[k+k\left(1+\beta k^{2}\right)\right] e^{-k n}-\left[s c-k\left(1+\beta k^{2}\right)\right] \frac{e^{-k(h-n)}}{\cosh k h}\right] \cos \\
\phi^{\prime}={ }_{n !}^{c} f_{0}^{\infty} \frac{k^{n}}{\Delta}\left[e^{-k(h-r)}+(-1)^{n+1} e^{k(h-n)}\right] e^{k y} \cos k x d k,
\end{array}
\end{aligned}
$$

which have the outgoing waves

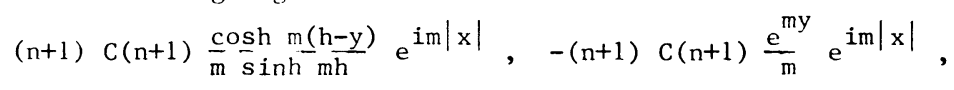

as $|x| \rightarrow \infty$, where $C(n)$ is given by (5.4), and when the singularity is at $(0,-n)$ in the upper fluid,

$$
\begin{aligned}
& \phi=\frac{2 s c}{n !} f_{0}^{\infty} \frac{k^{n}}{\Delta} e^{-k n \cosh k(h-y) \cos k x d k,} \\
& \phi^{\prime}=\frac{\cos (n+1) \theta}{R^{n+1}}+\frac{1}{n !} f_{0}^{\infty} \frac{k^{n}}{\Delta} e^{-k n\left[c(\cosh k h-s \sinh k h)-k\left(1+\beta k^{2}\right) \sinh k h\right] x} \\
& x e^{k y} \cos k x d k \text {, }
\end{aligned}
$$

and have the outgoing waves

$$
(n+1) C^{\prime}(n+1) \frac{\cosh m(h-y)}{m \sinh m h} e^{i m|x|},-(n+1) \quad C^{\prime}(n+1) \frac{e^{m y}}{m} e^{i m|x|},
$$

as $|x|+\infty$, where $C^{\prime}(n)$ is given by (5.11).

(b) Point singularities

For a singularity in the lower fluid,

$$
\phi=\frac{P_{n}(\cos \theta)}{R^{n+1}}+\frac{1}{n !} \int_{0}^{\infty} k^{n} e^{-k(h-n)} \frac{\sinh k y}{\cosh k h} J_{0}(k r) d k-\frac{1}{n !} f_{0}^{\infty} \frac{k^{n}}{\Delta}\left[(-1)^{n}\left[k+k\left(1+\beta k^{2}\right)\right]\right.
$$




$$
\begin{array}{r}
\left.-\left[s c-k\left(1+\beta k^{2}\right)\right] \frac{e^{-k(h-n)}}{\cosh k h}\right] \cosh k(h-y) J_{0}(k r) d k, \\
\phi^{\prime}=\frac{c}{n !} \int_{c}^{f^{\infty}} \frac{k^{n}}{\Delta}\left[e^{-k(h-n)}+(-1)^{n} e^{k(h-n)}\right] e^{k y} J_{0}(k r) d k,
\end{array}
$$

with the outgoing waves

$$
C(n) \frac{\cosh m(h-y)}{m \sinh m h} H_{0}^{(1)}(m r),-C(n) \frac{e^{m y}}{m} H_{0}^{(1)}(m r)
$$

as $r \rightarrow \infty, C(n)$ being given by $(5.4)$.

When the singularity is in the upper fluid,

$$
\begin{aligned}
\phi & =\frac{2 s c}{n !} \int_{0}^{\infty} \frac{k^{n}}{\Delta} e^{-k n} \cosh k(h-y) J_{0}(k r) d k \\
\phi^{\prime} & =\frac{P_{n}(\cos \theta)}{R^{n+1}}+\frac{1}{n !} \int_{0}^{\infty} \frac{k^{n}}{\Delta} e^{-k n}\left[c(\cosh k h-s \sinh k h)-k\left(1+B k^{2}\right) \sinh k h\right] \times \\
& \times e^{k y} J_{0}(k r) d k
\end{aligned}
$$

and as $r \rightarrow \infty, \phi, \phi^{\prime}$ have the outgoing waves

$$
C^{\prime}(n) \frac{\cosh m(h-y)}{m \sinh m h} H_{,}^{(1)}(m r), \quad-C(n) \frac{e^{m y}}{m} H_{o}^{(1)}(m r) \text {, }
$$

$C^{\prime}(n)$ being given by $(5.11)$.

6. SUBMERGED SINGULARITIES. BOTH FLUIDS INFINITE.

Herealso the boundary value problem for the velocity potentials $\phi, \phi^{\prime}$ is similar to the corresponding ones in sections 3,4 except that conditions (2.2) and (2.3) are replaced by

$$
\begin{aligned}
& \nabla \phi \rightarrow 0 \text { as } \mathrm{y} \rightarrow \infty, \\
& \nabla \phi^{\prime} \rightarrow 0 \text { as } \mathrm{y} \rightarrow-\infty
\end{aligned}
$$

respectively, and the radiation condition takes the forms

$$
\phi \sim C e^{-m y} e^{i m|x|}, \quad \phi^{\prime} \sim-C e^{m y} e^{i m|x|}
$$

as $|x| \rightarrow \infty$, for line singularities, and the forms

$$
\phi \sim \mathrm{Ce}^{-\mathrm{my}} \mathrm{H}_{\mathrm{o}}^{(1)}(\mathrm{mr}), \quad \phi^{\prime} \sim-\mathrm{Ce}^{\mathrm{my}} \mathrm{H}_{\mathrm{o}}^{(1)}(\mathrm{mr})
$$

as $r \rightarrow \infty$, for point singularities, where $C$ is a constant multiplier and $m$ is now the simple zero of the equation

$$
k\left(1+B k^{2}\right)-c(1+s)=0
$$

The evaluation of $\phi$, $\phi^{\prime}$ for each singularity can be carried out independently (see [5]). They may also be evaluated by letting $h$ in the results of the previous section tend formally to infinity. The velocity potentials for the different singularities are as follows:

(a) Line singularities.

(i) Wave source.

The velocity potentials are

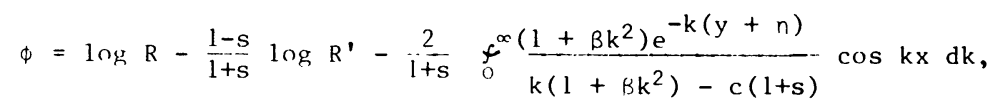

$$
\phi^{\prime}=\frac{2}{1+s} \log R+\frac{2}{1+s}{\underset{0}{f^{\infty}}}_{\frac{\left(1+\beta k^{2}\right) e^{k(y-n)}}{k\left(1+\beta k^{2}\right)-c(1+s)}} \cos k x d k,
$$

for a wave source in the lower fluid and 


$$
\begin{aligned}
& \phi^{\prime}=\frac{2 s}{1+s} \log R+\frac{2 s}{1+s} f_{0}^{\infty} \frac{\left(1+\beta k^{2}\right) e^{-k(y+n)}}{k\left(1+\beta k^{2}\right)-c(1+s)} \cos k x d k, \\
& \phi^{\prime}=\log R+\frac{1-s}{1+s} \log R^{\prime}-\frac{2 s}{1+s} f_{0}^{\infty} \frac{\left(1+\beta k^{2}\right) e^{k(y-n)}}{k\left(1+\beta k^{2}\right)-c(1+s)} \cos k x d k,
\end{aligned}
$$

for a wave source in the upper fluid.

(ii) Multipoles singularities.

The velocity potentials are

$$
\begin{aligned}
& \phi=\frac{\cos (n+1) \theta}{R^{n+1}}+\frac{(-1)^{n+1}}{n !} \underbrace{f^{\infty}}_{0} \frac{\left[k\left(1+\beta k^{2}\right)+k\right] k^{n} e^{-k\left(y+r_{1}\right)}}{k\left(1+\beta k^{2}\right)-c(1+s)} \cos k x d k, \\
& \phi^{\prime}=\frac{2(-1)^{n} c}{n !} \int_{0}^{\infty} \frac{k^{n} e^{k(y-n)}}{k\left(1+3 k^{2}\right)-c(1+s)} \cos k x d k,
\end{aligned}
$$

if the singularity is in the lower fluid and

$$
\begin{aligned}
& \phi=\frac{-2 s c}{n !} f_{0}^{\infty} \frac{k^{n} e^{-k(y+n)}}{k\left(1+\beta k^{2}\right)-c(1+s)} \cos k x d k, \\
& \phi^{\prime}=\frac{\cos (n+1) \theta}{R^{n+1}}+\frac{1}{n !} \int_{0}^{\infty} \frac{k^{n}\left[k\left(1+\beta k^{2}\right)-k\right]}{k\left(1+\beta k^{2}\right)-c(1+s)} e^{k(y-n)} \cos k x d k, \\
& \text { if the singularity is in the upper fluid. }
\end{aligned}
$$

(b) Point singularities

If the singularity is in the lower fluid

$$
\begin{aligned}
& \phi=\frac{P_{n}(\cos \theta)}{R^{n+1}}+\frac{(-1)^{n}}{n !} f_{0}^{\infty} \frac{\left[k\left(1+\beta k^{2}\right)+k\right] k^{n} e^{-k(y+n)}}{k\left(1+\beta k^{2}\right)-c(1+s)} J_{0}(k r) d k, \\
& \phi^{\prime}=\frac{2 c(-1)^{n+1}}{n !} f_{0}^{\infty} \frac{k^{n} e^{k(y-n)}}{k\left(1+\beta k^{2}\right)-c(1+s)} J_{0}(k r) d k
\end{aligned}
$$

and if it is in the upper fluid,

$$
\begin{aligned}
& \phi=\frac{-2 s c}{n !} f_{0}^{\infty} \frac{k^{n} e^{-k(y+n)}}{k\left(1+B k^{2}\right)-c(1+s)} J_{0}(k r) d k, \\
& \phi^{\prime}=\frac{P_{n}(\cos \theta)}{R^{n+1}}+\frac{1}{n !} f_{0}^{\infty} \frac{k^{n}\left[k\left(1+B k^{2}\right)-K\right]}{k\left(1+B k^{2}\right)-c(1+s)} e^{k(y-n)} J_{0}(k r) d k .
\end{aligned}
$$

7. SINGULARITIES AT THE SURFACE OF SEPARATION.

Clearly the results of the previous sections are not valid for $n=0$. Here we use coordinates based on the singularity at the urgin. Then it may be shoen that the potentials are as follows

(a) Line singularities

(i) Wave source

Both fluids of finite depth

$$
\left.\begin{array}{l}
\phi=y_{0}^{\infty} \frac{1}{k \Delta}\left[2 c s+\left(k\left(1+\beta k^{2}\right) \sinh k h^{\prime}-2 c s \cosh k h^{\prime}\right) \cosh k(h-y) \cos k x\right] d k, \\
\phi^{\prime}=\int_{0}^{\infty} \frac{1}{k \Delta}\left[2 c+\left(k\left(1+\beta k^{2}\right) \sinh k h-2 c \cosh k h\right) \cosh k^{\prime}\left(h^{\prime}+y\right) \cos k x\right] d k
\end{array}\right\}
$$

where $\Delta$ is given by (3.2) . 
Lower fluid of finite depth

$\phi=2 s \log R+f_{0}^{\infty} \frac{\delta}{L}\left(1+\beta k^{\prime}\right)(\cosh k h-s \sinh k h) \cosh k(h-y) \cos k x d k$

$$
+2 s \int_{0}^{\infty} \frac{1}{k}\left[e^{-k y}-\delta \cosh k(h-y)\right] \cos k x d k \text {, }
$$

$\phi^{\prime}=2 \log R-f_{0}^{\infty} \frac{\delta}{\Delta}\left(1+\beta k^{2}\right)(\cosh k h-s \sinh k h) \sinh k h e^{k y} \cos k x d k$

$$
+2 s \int_{0}^{\infty} \frac{\delta}{k} \sinh k h e^{k y} \cos k x d k
$$

where $\delta^{-1}=\cosh k h+s \sinh k h$, and $\Delta$ is given by $(5.1)$.

Both fluids infinite

$$
\left.\begin{array}{l}
\phi=\frac{2 s}{1+s} \log R-\frac{2}{1+s} f_{0}^{\infty} \frac{\left(1+\beta k^{2}\right) e^{-k y}}{k\left(1+\beta k^{2}\right)-c(1+s)} \cos k x d k, \\
\phi^{\prime}=\frac{2}{1+s} \log R+\left(\frac{1-s}{1+s}\right) f_{0}^{\infty} \frac{\left(1+\beta k^{2}\right) e^{k y}}{k\left(1+\beta k^{2}\right)-c(1+s)} \cos k x d k,
\end{array}\right\}
$$

(ii) Multipoles

Both fluid of finite depth

For multipoles corresponding to $\mathrm{n}=1,3,5, \ldots$ (even multipoles)

$\left.\phi=\frac{1}{(2 m+1) !}{\underset{0}{0}}_{0}^{\infty} \frac{k^{2 m+1}}{\Delta}\left[2 s c \cosh k h^{\prime}-k\left(1+\beta k^{2}\right) \sinh k h^{\prime}\right] \cosh k(h-y) \cos k x d k,\right)$

$\left.\phi^{\prime}=\frac{1}{(2 m+1) !} \quad f_{0}^{\infty} \frac{k^{2 m+1}}{\Delta}\left[2 c \cosh k h-k\left(1+\beta k^{2}\right) \sinh k h\right] \cosh k\left(h^{\prime}+y\right) \cos k x d k,\right\}$ and for the multipoles corresponding to $\mathrm{n}=0,2,4, \ldots$ (odd multipoles)

$\phi=\frac{\mathrm{c}(1+\mathrm{s})}{(2 \mathrm{~m}) !}{\underset{0}{0}}^{\infty} \frac{\mathrm{k}^{2 \mathrm{~m}}}{\Delta} \sinh \mathrm{kh}^{\prime} \cosh \mathrm{k}(\mathrm{h}-\mathrm{y}) \cos \mathrm{kx} \mathrm{dk}$,

$\left.\phi^{\prime}=\frac{-c(1+s)}{(2 m) !} f_{0}^{\infty} \frac{k^{2 m}}{\Delta} \sinh k h \cosh k\left(h^{\prime}+y\right) \cos k x d k,(m=0,1,2, \ldots)\right\}$

where $\Delta$ is given by (3.2).

Lower fluid of finite depth

Similarly for even multipoles $(m=0,1,2, \ldots)$

$\phi=\frac{1}{(2 m+1) !} f_{0}^{\infty} \frac{k^{2 m+1}}{\Delta}\left[2 s c-k\left(1+\beta k^{2}\right)\right] \cosh k(h-y) \cos k x d k$,

$\left.\phi^{\prime}=\frac{1}{(2 m+1) !} f_{0}^{\infty} \frac{k^{2 m+1}}{\Delta}\left[2 c \cosh k h-k\left(1+B k^{2}\right) \sinh k h\right] e^{k y} \cos k x d k,\right\}$

and for odd multipoles

$\left.\begin{array}{l}\phi=\frac{c(1+s)}{(2 m) !} f_{0}^{\infty} \frac{k^{2 m}}{\Delta} \cosh k(h-y) \cos k x d k, \\ \phi^{\prime}=\frac{-c(1+s)}{(2 m) !} f_{0}^{\infty} \frac{k^{2 m}}{\Delta} \text { sinh } k h e^{k y} \cos k x d k,\end{array}\right\}$

where $\Delta$ is given by $(5.1)$.

Both fluids infinite

For multipoles corresponding to $n=1,3,5, \ldots$ (even multipoles), we have $\left.\begin{array}{l}\phi=\frac{1}{(2 m+1) !} f_{0}^{\infty} \frac{k^{2 m+1}\left[k\left(1+\beta k^{2}\right)-2 s c\right] e^{k y}}{k\left(1+\beta k^{2}\right)-c(1+s)} \cos k x d k, \\ \phi^{\prime}=\frac{1}{(2 m+1) !} f_{0}^{\infty} \frac{k^{2 m+1}\left[k\left(1+\beta k^{2}\right)-2 c l e^{m y}\right.}{1 k\left(1+\beta k^{2}\right)-c(1+s)} \cos k x d k,\end{array}\right\}$ 
and for odd multipoles

$$
\left.\begin{array}{l}
\phi=\frac{-c(1+s)}{(2 m) !} \quad f_{0}^{\infty} \frac{k^{2 m} e^{-k y}}{k\left(1+B k^{2}\right)-c(1+s)} \cos k x d k, \\
\phi^{\prime}=\frac{c(1+s)}{(2 m) !} \quad f_{0}^{\infty} \frac{k^{2 m}}{k\left(1+B k^{2}\right)-c(1+s)} \cos k x d k, \quad(m=0,1,2, \ldots)
\end{array}\right\}
$$

(b) Point singularities

Both fluids of finite depth

For multipoles corresponding to $n=0,2,4, \ldots$ (even multipoles)

$\left.\begin{array}{l}\phi=\frac{1}{(2 m) !}{\underset{0}{0}}_{0}^{\infty} \frac{k^{2 m}}{\Delta}\left[2 s c \cosh k h^{\prime}-k\left(1+B k^{2}\right) \sinh k^{\prime}\right] \cosh k(h-y) J_{0}(k r) d k, \\ \phi^{\prime}=\frac{1}{(2 m) !}{\underset{0}{f}}_{0}^{\infty} \frac{k^{2 m}}{\Delta}\left[2 s c \cosh k h-k\left(1+B k^{2}\right) \sinh k h\right] \cosh k\left(h^{\prime}+y\right) J_{0}(k r) d k,\end{array}\right\}$

and for multipoles corresponding to $n=1,3,5, \ldots$ (odd multipoles)

$$
\left.\begin{array}{l}
\phi=\frac{c(1+s)}{(2 m+1) !} f_{0}^{\infty} \frac{k^{2 m+1}}{\Delta} \sinh k h^{\prime} \cosh k(h-y) J_{0}(k r) d k, \\
\phi^{\prime}=\frac{-c(1+s)}{(2 m+1) !} f_{0}^{\infty} \frac{k^{2 m+1}}{\Delta} \text { sinh } k h \cosh k\left(h^{\prime}+y\right) J_{0}(k r) d k, \quad(m=0,1,2, \ldots)
\end{array}\right\}
$$

where $\Delta$ is given by (3.2).

\section{Lower fluid of finite depth}

For even multipoles $(m=0,1,2, \ldots)$

$$
\left.\begin{array}{l}
\phi=\frac{1}{(2 m) !}{\underset{f}{\infty}}^{\infty} \frac{k^{2 m}}{\Delta}\left[2 s c-k\left(1+\beta k^{2}\right)\right] \cosh k(h-y) J_{0}(k r) d k, \\
\phi^{\prime}=\frac{1}{(2 m) !}{\underset{0}{\infty}}^{\infty} \frac{k^{2 m}}{\Delta}\left[2 c \cosh k h-k\left(1+\beta k^{2}\right) \sinh k h\right] e^{k y} \cos k x d k,
\end{array}\right\}
$$

and for odd multipoles

$$
\left.\begin{array}{l}
\phi=\frac{c(1+s)}{(2 m+1) !} f_{0}^{\infty} \frac{k^{2 m+1}}{\Delta} \cosh k(h-y) J_{0}(k r) d k \\
\phi^{\prime}=\frac{-c(1+s)}{(2 m+1) !} f_{0}^{\infty} \frac{k^{2 m+1}}{\Delta} \sinh k h e^{k y} J_{0}(k r) d k,
\end{array}\right\}
$$

where $\Delta$ is given by (5.1).

Both fluids infinite

For multipoles corresponding to $n=0,2,4, \ldots$ (even multipoles), we have

$$
\left.\begin{array}{l}
\phi=\frac{1}{(2 m) !}{\underset{0}{0}}_{0}^{\infty} k^{2 m} \frac{\left[k\left(1+\beta k^{2}\right)-2 s c\right] e^{k y}}{k\left(1+B k^{2}\right)-c(1+s)} J_{0}(k r) d k, \\
\phi^{\prime}=\frac{1}{(2 m) !} \int_{0}^{f^{\infty}} k^{2 m} \frac{\left[k\left(1+\beta k^{2}\right)-2 c\right] e^{-k y}}{k\left(1+B k^{2}\right)-c(1+s)} J_{0}(k r) d k,
\end{array}\right\}
$$

and for odd multipoles

$$
\left.\begin{array}{l}
\phi=\frac{-c(1+s)}{(2 m+1)} \quad f_{0}^{f^{\infty}} k^{2 m+1} \frac{e^{k y}}{k\left(1+\beta k^{2}\right)-c(1+s)} J_{\circ}(k r) d k, \\
\phi^{\prime}=\frac{c(1+s)}{(2 m+1)} \quad f_{0}^{f^{\infty} k^{2 m+1}} \frac{e^{-k y}}{k\left(1+\beta k^{2}\right)-c(1+s)} J_{\circ}(k r) d k . \quad(m=0,1,2, \ldots) .
\end{array}\right\}
$$


It should be noted here that there is a non-uniqueness for $\beta>0$ to the extent that any multiple of a slope potential may be added. The forms given above correspond to a continuous interface slope tt the orgin, where the interface elevation is always finite.

8. CONCLUSION.

A complete survey for all the basic singularities that can be used in two fluids problems with surface tension is presented. Results of Gorgui and Kaseem [2] and Kaseem [3] can be recovered by putting $\beta=0$ in the appropriate forms and also those of Rhodes-Robinson [4] can be obtained by putting $s=o$.

\section{REFERENCES}

1. GORCiUI, M. A. Wave motion due to a cylinder heaving at the surface separating two infinite liquids. Jour. Nat. Sc. Math. XVI (1976), 1-20.

2. GORGUI, M.A. and KASEEM, S.E. Basic singularities in the theory of internal waves. Quart. J. Mech. Appl. Math. 31 (1978), 31-48.

3. KASEEM, S. E. Multipole expansions for two superposed fluids, each of finite depth. Proc. Camb. Phil. Soc. 91 (1982), 323-329.

4. RHODES-ROBINSON, P. F. Fundamental singularities in the theory of water waves-with surface tension. Bul1. Austral. Math. Soc. 21(1970), 317-333.

5. FALTAS, M. S. Internal waves in two-fluids problems. M. Sc. Thesis. University of Alexandria, Egypt, (1977). 


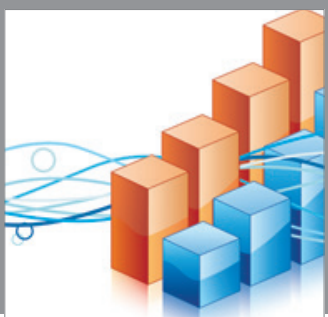

Advances in

Operations Research

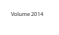

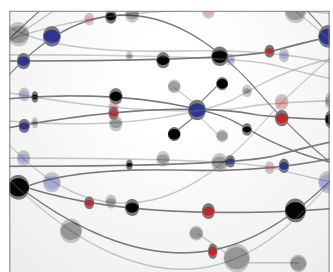

\section{The Scientific} World Journal
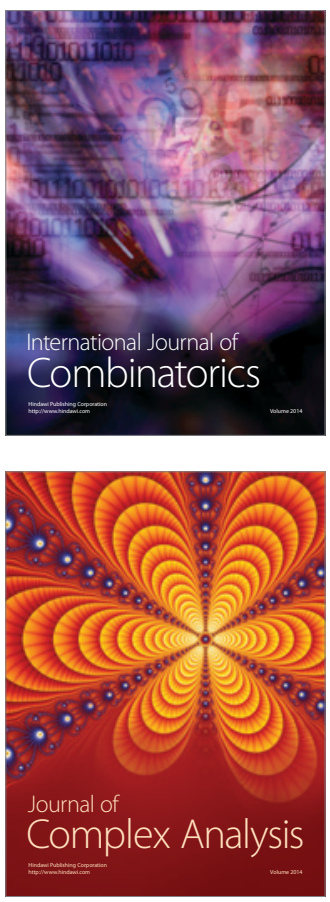

International Journal of

Mathematics and

Mathematical

Sciences
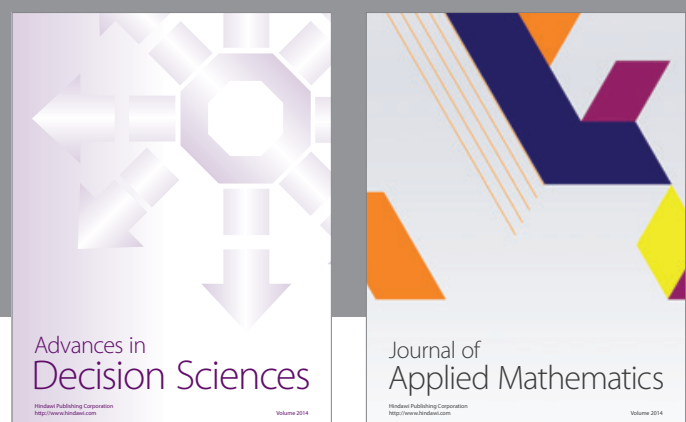

Journal of

Applied Mathematics
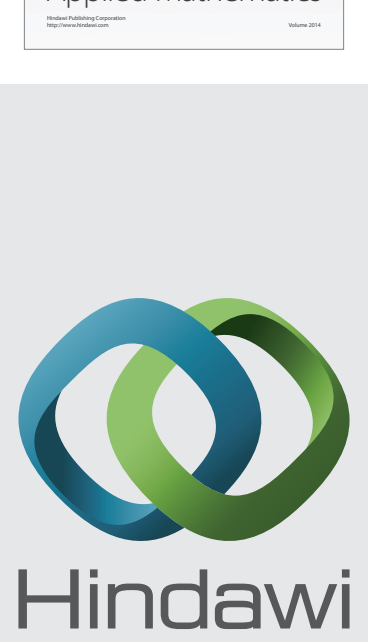

Submit your manuscripts at http://www.hindawi.com
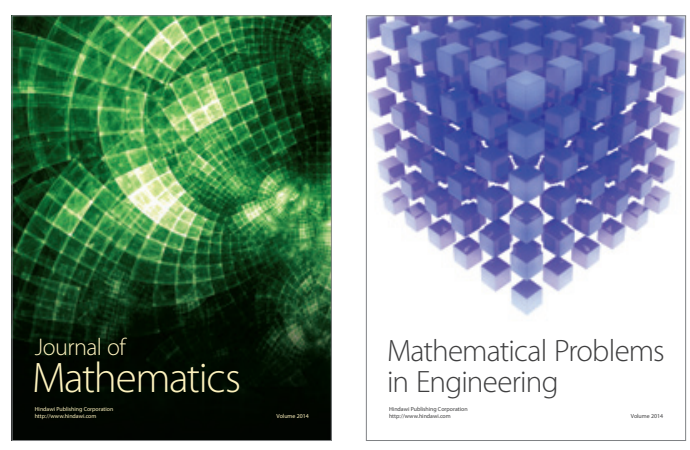

Mathematical Problems in Engineering
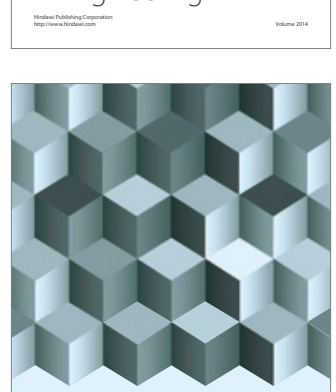

Journal of

Function Spaces
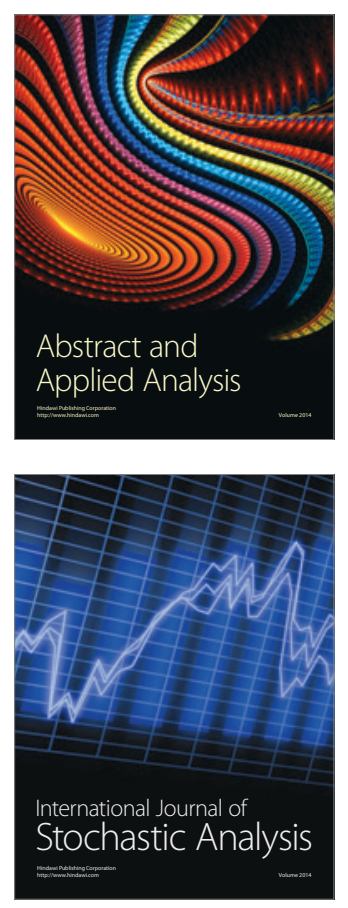

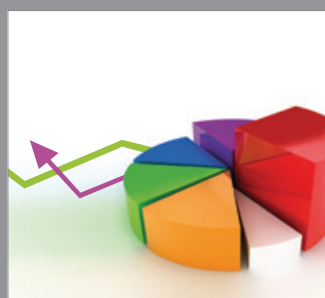

ournal of

Probability and Statistics

Promensencen
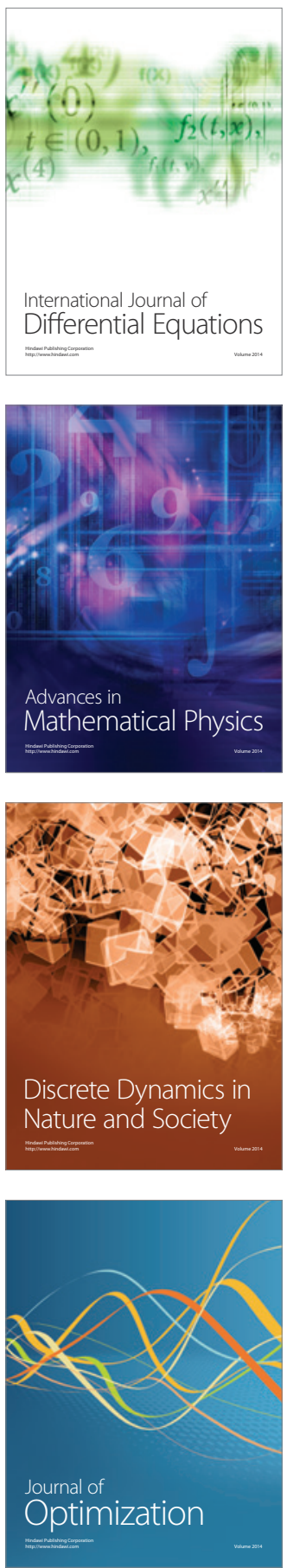